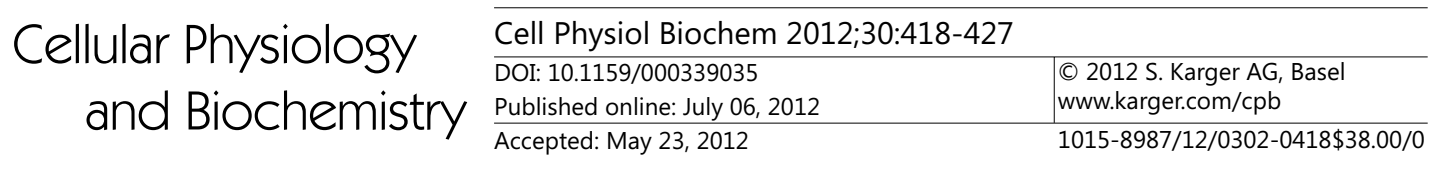

\title{
Red Blood Cell Alterations in Systemic Sclerosis: a Pilot Study
}

\author{
Antonello Giovannetti ${ }^{1}$ Lucrezia Gambardella ${ }^{2}$ Donatella Pietraforte ${ }^{3}$ Edoardo \\ Rosato $^{1}$ Anna Maria Giammarioli ${ }^{2}$ Felice Salsano ${ }^{1}$ Walter Malorni ${ }^{2,4, *}$ Elisabetta \\ Straface ${ }^{2 *}$
}

${ }^{1}$ Department of Clinical Medicine, Clinical Immunology Unit-Scleroderma Center, Sapienza University of Rome, Rome; ${ }^{2}$ Department of Therapeutic Research and Medicine Evaluation, Istituto Superiore di Sanità, Rome, Rome; ${ }^{3}$ Department of Cell Biology and Neuroscience, Istituto Superiore di Sanità, Rome, Rome; ${ }^{1}$ San Raffaele Institute Sulmona, L'Aquila, *to be considered as senior authors

\author{
Key Words \\ Oxidative stress • Erythrocytes • Scleroderma
}

\begin{abstract}
Aims: The aim of this work was to investigate whether systemic oxidative imbalance that occurs in patients with systemic sclerosis affects red blood cell integrity. Methods: Reactive oxygen species, intracellular content of total thiols and molecules involved in red blood cell aging (e.g., glycophorin A, band 3, CD47 and phosphatidylserine externalization), have been analyzed in erythrocytes from 39 patients with systemic sclerosis and 30 healthy donors by using flow and static cytometry. Analyses were carried out taking into account the two clinical subsets of scleroderma: diffuse cutaneous sclerosis and limited cutaneous sclerosis. Results: A significant reduction $(p<0.05)$ of intracellular total thiols and a significant loss $(p<0.01)$ of glycophorin A, band 3 and CD47 was found in red blood cells from patients with limited cutaneous sclerosis. Conversely, a significant increase $(p<0.01)$ of reactive oxygen species levels and CD47 expression was found in red blood cells from patients with diffuse cutaneous sclerosis. Phosphatidylserine externalization was significantly increased both in patients with limited and diffuse disease. Importantly, this increase was related with disease severity and nailfold capillaroscopy. Conclusions: Altogether these results suggest a reappraisal of the red blood cells as useful markers in the clinical management of the disease.
\end{abstract}




\begin{tabular}{|c|c|c|}
\hline \multirow{2}{*}{$\begin{array}{l}\text { Cellular Physiology } \\
\text { and Biochemistry }\end{array}$} & \multicolumn{2}{|c|}{ Cell Physiol Biochem 2012;30:418-427 } \\
\hline & $\begin{array}{l}\text { DOI: } 10.1159 / 000339035 \\
\text { Published online: July 06, } 2012\end{array}$ & $\begin{array}{l}\text { O } 2012 \text { S. Karger AG, Basel } \\
\text { www.karger.com/cpb }\end{array}$ \\
\hline
\end{tabular}

\section{Introduction}

Scleroderma or systemic sclerosis (SSc) is a rare $(0.1 \%$ of the US population and 2.5 million persons worldwide) acquired autoimmune disorder characterized by microvascular dysfunction, increased deposition of extracellular matrix in the skin and internal organs, and Raynaud's phenomenon (RP) [1, 2]. The disease most commonly affects adult women, and its progression and severity vary greatly among different individuals with death occurring as a result of end-stage organ failure. Two clinical subsets of scleroderma are now widely accepted: limited cutaneous SSc (lc-SSc) and diffuse cutaneous SSc (dc-SSc), generally considered as the more severe subset of the disease [3]. The main difference between these two subsets is the speed of disease progression and the extent and severity of skin and visceral involvement. Limited cutaneous SSc has an insidious onset with skin involvement confined largely to the face and extremities whereas patients in both subsets can develop ischemic digital ulcers. Profound small-vessel remodeling was hypothesized as more pronounced in lc-SSc. However, the occurrence of pulmonary arterial hypertension $(\mathrm{PAH})$, has been observed either in patients with lc-SSc or in patients with dc-SSc $[4,5]$. There is consistent evidence that oxidative stress, the shift of balance between reactive oxygen species (ROS) generation and an efficient system of antioxidants in favor of ROS production, is associated with SSc but, until now, its specific involvement in the pathogenesis of vascular injury and fibrosis or in clinical manifestation of SSc remains unclear [6]. The RP and the chronic inflammatory process, which generally occur in SSc patients, could partially be responsible for ROS generation. In fact, several studies point out that monocytes from patients with SSc spontaneously release higher levels of superoxide anion than monocytes from patients with primary RP and from healthy donors [7]. Moreover, there is convincing evidence that ROS and their targets [e.g., low-density lipoproteins (LDL) that are modified to oxidized-LDL] are involved in endothelial cell damaging and intimal thickening [8, 9]. ROS can stimulate fibroblast proliferation and extracellular matrix protein production, suggesting that oxidative imbalance can be implicated in the pathogenesis of the SSc fibrosing process [10]. On the basis of this evidence, a study has been undertaken on the peripheral blood of SSc patients with the aim to investigate if oxidative stress, which occurs in these patients, affects red blood cell (RBC) integrity.

RBCs are peculiar cells aimed at the delivery of oxygen and nitric oxide to the periphery and carbon dioxide to the lungs $[11,12]$. In addition, they also exert, under physiological conditions, a scavenging activity towards reactive oxygen and nitrogen species often overproduced in morbidity states, e.g. in inflamed tissues. Their deformability is essential for their circulation, specifically in small blood vessels, and this is an important pre-requisite for such vascular "antioxidant" functions. Conversely, changes in the redox state result in subvertion of RBC function: they become a source of reactive species. Furthermore, this is associated with the loss of RBC typical structural features [13], increased aggregability [14] and adhesiveness to the endothelium and to other blood cells [15], thus contributing to vascular damage.

\section{Materials and Methods}

\section{Study population}

Thirty-nine consecutive outpatients (32 females and 7 males) fulfilling the American College of Rheumatology criteria for SSc [16] were enrolled for the study, with their informed consent and institutional approval. Patients were classified as having limited cutaneous SSc (lc-SSc, $n=20$ ) or diffuse cutaneous SSc (dc-SSc, $n=19$ ) on the basis of the criteria of LeRoy et al. [3]. Thirty healthy donors (HD) were used as controls. In Table 1 clinical features of the study population are listed. Exclusion criteria were: previous or concomitant treatments with immunosuppressive drugs and therapy with endothelin inhibitors. Patients who were receiving steroids ( $\mathrm{n}=5$, prednisone $\leq 5 \mathrm{mg} /$ day) discontinued the treatment 30 days before immunological assays. The study was approved from the local Ethical Committee, and all patients and control subjects gave written informed consent. 


\section{Cellular Physiology and Biochemistry}

Cell Physiol Biochem 2012;30:418-427

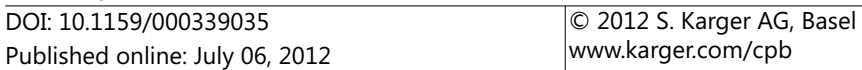

Giovannetti/Gambardella/Pietraforte et al.: Erythrocyte Fate in Scleroderma

\begin{tabular}{lcccc}
\hline \multicolumn{1}{c}{ Group } & Sex & $\begin{array}{c}\text { Age, median } \\
\text { (range) years }\end{array}$ & $\begin{array}{c}\text { Disease duration, } \\
\text { median (range) } \\
\text { Years }\end{array}$ & $\begin{array}{c}\text { Raynaud duration } \\
\text { (years) }\end{array}$ \\
\hline F/M & & $5(1-22)$ & $16.5(1-47)$ \\
dc-SSc & $17 / 3$ & $57(31-73)$ & $7.5(2-16)$ & $10.5(4-17)$ \\
Healthy donors & $15 / 4$ & $47(22-75)$ & - & - \\
\hline
\end{tabular}

Table 1. Clinical features of SSc patients and healthy donors.

Clinical assessment

In each patient the organ involvement was exhaustively investigated. Skin induration was assessed by modified Rodnan Total Skin Score (mRTSS) method. It was determined at a standardized location of 17 different sites of the body with a standardized pinching method and it is scored from 0 to 3 [17]. Therefore mRTSS value significantly correlates with disease activity index in univariate analysis [18]. Disease activity was also measured using Valentini's Scleroderma Disease Activity Score (SDAS), which consists of 10 weighted variables: total skin score $>14$, scleroderma, digital necrosis, arthritis, total lung capacity $<80 \%$, erythrocyte sedimentation rate (ESR) $>30$, hypocomplementemia and change in cardiopulmonary, skin and vascular symptoms in the past month [18, 19]. Finally, disease severity was measured using Medsger Scleroderma Disease Severity Scale (DSS). The original scale assessed disease severity in 9 organs or systems, namely general health, peripheral vascular, skin, joint/tendon, muscle and gastrointestinal tract, lungs, heart and kidneys. Each organ/system is scored separately from 0 to 4 depending on whether there is no, mild, moderate, severe or end-stage involvement [20].

Nailfold videocapillaroscopy (NVC)

NVC was performed by single operator using a videocapillaroscope (Pinnacle Studio Version 8) equipped with a $500 \times$ optical probe. NVC was performed after resting the subject in a temperature controlled room at $24 \pm 0,4{ }^{\circ} \mathrm{C}$ for $20 \mathrm{~min}$. The nailfold of the second, third, fourth and fifth finger was examined in each patient. Microvascular alterations, as detected by NVC in patients with SSc, have been classified in three different patterns according to Cutolo et al [21]. The patterns identified within the "SSc pattern" include: i) Early NVC pattern: few enlarged/giant capillaries, few capillary haemorrhages, relatively well-preserved capillary distribution and no evident loss of capillaries; ii) Active NVC pattern: frequent giant capillaries, frequent capillary haemorrhages, moderate loss of capillaries, mild disorganization of the capillary architecture and absent or mild ramified capillaries; iii) Late NVC pattern: irregular enlargement of the capillaries, few or absent giant capillaries and haemorrhages, severe loss of capillaries with extensive avascular areas, disorganization of the normal capillary array and ramified/bushy capillaries.

\section{Isolation of red blood cells}

Fresh human blood from healthy donors and SSc patients was drawn into heparinized tubes. For RBC isolation, whole blood was centrifuged for $10 \mathrm{~min}$ at $1,500 \mathrm{~g}$. The plasma and buffy coat were removed, RBCs were washed twice in isotonic phosphate-buffered saline (PBS), $\mathrm{pH} 7.4$, and resuspended in the same buffer to the initial hematocrit concentration. No appreciable cell lysis was observed during the RBC preparation procedure.

\section{Analysis of the redox balance}

For intracellular ROS production, RBCs ( $5 \times 10^{5}$ cells) were incubated in Hanks' balanced salt solution (HBSS, pH 7.4) containing dihydrorhodamine 123 (DHR 123, Molecular Probes, USA). Intracellular content of total thiols was explored by using 5-chloromethylfluoresceindiacetate (CMFDA, Molecular Probes). Samples were then analyzed with a FACScan flow cytometer (Becton Dickinson, Mountain View, CA, USA). The median values of fluorescence intensity histograms were used to provide semi-quantitative evaluation of reduced thiols content and ROS production.

\section{Evaluation of RBC injury}

Quantitative evaluation of RBC "apoptosis", was performed by flow cytometry after double staining method using FITC-conjugated Annexin V apoptosis detection kit (Woods Hole Marine Biological Laboratory, 


\section{Cellular Physiology and Biochemistry}

Cell Physiol Biochem 2012;30:418-427

\begin{tabular}{l|l}
\hline DOI: $10.1159 / 000339035$ & C 2012 S. Karger AG, Basel
\end{tabular}

Published online: July 06, 2012

www.karger.com/cpb

Giovannetti/Gambardella/Pietraforte et al.: Erythrocyte Fate in Scleroderma

\begin{tabular}{lcccc}
\hline & Healthy donors & All SSc patients & lc-SSc patients & dc-SSc patients \\
\hline ROS levels & $56 \pm 3$ & $67 \pm 6^{*}$ & $61 \pm 4^{*}$ & $74 \pm 3^{* *}$ \\
Total thiols & $53 \pm 2$ & $49 \pm 6$ & $42 \pm 5^{*}$ & $55 \pm 5$ \\
\hline
\end{tabular}

Table 2. Redox balance in red blood cells. The numbers represent the mean of median fluorescence intensity. To compare healthy donors with the two subgroups of SSc patients, one-way ANOVA followed by Tukey Test was used. We found significant differences between the three subgroups (ROS: $<0.0001$; Total thiols: $\mathrm{p}=0.0012) .\left(^{*}\right) \mathrm{p}<0.05$ vs healthy donors; $\left({ }^{* *}\right) \mathrm{p}<0.0001$ vs healthy donors.

USA) and $0.05 \%$ Trypan blue for $10 \mathrm{~min}$ at room temperature. Samples were analyzed by flow cytometry with a FACScan flow cytometer (Becton Dickinson, Mountain View, CA, U.S.A.) equipped with a 488-nm argon laser.

\section{Analytical cytology}

RBCs were fixed with $3.7 \%$ formaldehyde in PBS (pH 7.4) for $10 \mathrm{~min}$ at room temperature, and washed in the same buffer. They were then permeabilized with $0.5 \%$ Triton X-100 in PBS for 5 min at room temperature. After washing with PBS, samples were stained with: monoclonal anti-glycophorin A (Sigma-Aldrich, St Louis, US), monoclonal anti-band 3 (Sigma), and monoclonal anti-CD47 (Santa Cruz Biotechnology, San Diego, CA). After $30 \mathrm{~min}$ at $37^{\circ} \mathrm{C}$, samples were washed and then incubated for $30 \mathrm{~min}$ at $37^{\circ} \mathrm{C}$ with fluorescein isothiocyanate (FITC)-labeled anti mouse (Sigma). Secondary antibody given alone was used as negative control. Samples were analyzed by an Olympus BX51 Microphot fluorescence microscope or by flow cytometry with a FACScan flow cytometer (Becton Dickinson, Mountain View, CA, U.S.A.) equipped with a 488-nm argon laser. At least 20,000 events have been acquired. The median values of fluorescence-intensity histograms were used to provide semiquantitative analysis. The values of the untreated RBCs were set to $100 \%$, and the levels of the considered proteins in the different samples were reported relative to these.

\section{Statistical analysis}

Normally distributed data were expressed as mean \pm standard deviation. In order to compare control group with the two subgroups of SSc patients (limited and diffuse), one-way ANOVA was used, followed by Tukey Test by using Prism 4 Graphpad software for MacIntosh.

Due to the small sample size, non-parametric tests were used for the analysis of clinical parameters. The Kruskal-Wallis test was used to test differences between more than 2 subgroups followed by the Bonferroni post-hoc test in case of significance. The data non normally distributed were expressed as median and range. Spearman's rank order correlation was used to test for an association between clinical variables (mRTSS, DSS and SDAS) and PS externalization. P-values $<0.05$ were considered as significant. Commercial software (SPSS version 18) was used for these statistical analyses.

\section{Results}

\section{Redox balance}

In order to verify if oxidative stress that occurs in SSc patients influences RBC morphology and function, two important parameters were analyzed. In particular, ROS production and intracellular content of total thiols, known to influence the redox balance of the cell, have been evaluated. As reported in Table 2, a significant increase of ROS levels $(\mathrm{p}<0.05)$ was detected in RBCs from SSc patients with respect to those from healthy donors. Interestingly, when the data obtained were analyzed taking into account of two clinical subsets of scleroderma, significantly higher levels of ROS have mainly been found in RBCs from patients with dc-SSc $(\mathrm{p}<0.01)$ with respect to those from healthy donors. Moreover, considering that a mild, but persistent oxidative stress, can lead to glutathione (GSH) depletion and to oxidation of thiol groups, a measurement of total thiols, essentially referred as to cytoskeletal thiol groups that oversee cell-shape maintenance [22], was performed by flow-cytometry. As shown in Table 2 a significant $(\mathrm{p}<0.05)$ reduction of the intracellular content of total thiols was detected in 


\section{Cellular Physiology Cell Physiol Biochem 2012;30:418-427

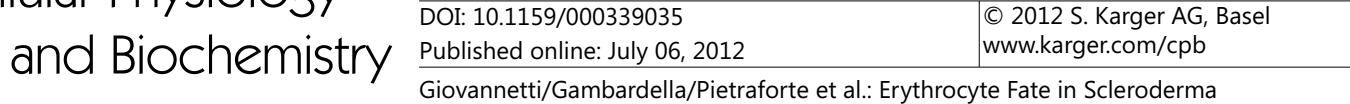

$$
\begin{aligned}
& \text { - Healthy donors } \\
& \square \text { Ic-SSc patients } \\
& - \text { dc-SSc patients }
\end{aligned}
$$

$\mathbf{A}$

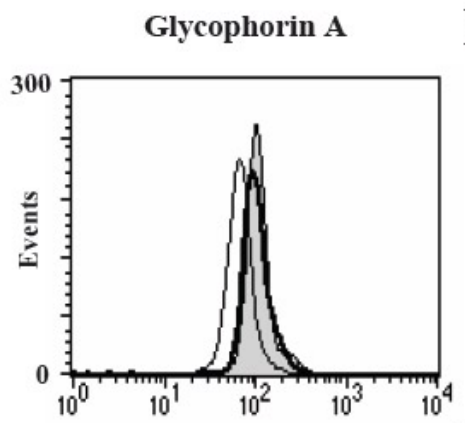

C

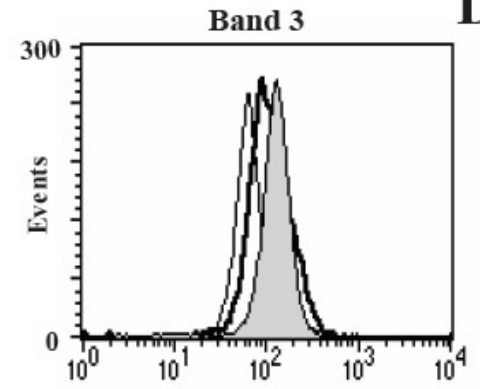

D
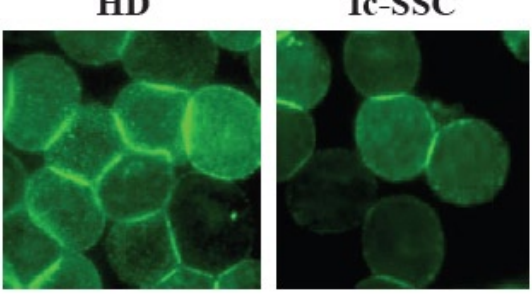

HD

Ic-SSC
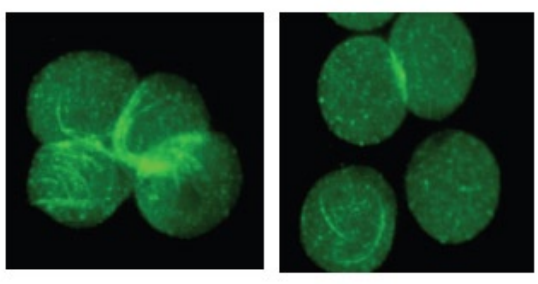

F

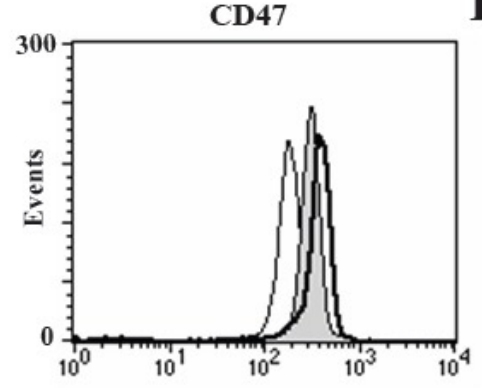

HD

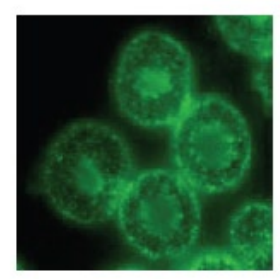

Ic-SSC

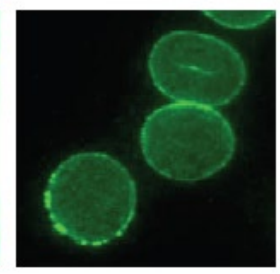

Fig. 1. Analysis of molecules involved in RBC aging. In left column are reported three histograms showing flow cytometry evaluation of: (A) GA; (C) band 3 and (E) CD47 in RBCs from a representative healthy donor, a representative lc-SSc patient and a representative dc-SSc patient. The expression of these molecules appears significantly lower in RBCs from lc-SSc patients $(\mathrm{p}<0.01)$. In right column are reported representative immunofluorescence micrographs displaying different arrangement and positivity of: GA (B); band 3 (D) and CD47 (F) in RBCs from one healthy donor and one lc-SSc patient.

RBCs from lc-SSc patients in comparison with RBCs from healthy donors, whereas values detected in RBCs from dc-SSc patients were substantially similar to those detected in RBCs from healthy donors.

\section{Aging markers}

Considering that an alteration of the redox status is associated with RBC aging and degeneration [23], four different "indicators" of senescence have been selected: glycophorin A (GA), band 3, CD47 and phosphatidylserine (PS) externalization. The first is a glycoprotein widely expressed at the RBC surface that is down-regulated during senescence. The second is an "anion channel molecule" involved in RBC homeostasis maintenance. The third is 


\section{Cellular Physiology and Biochemistry}

Cell Physiol Biochem 2012;30:418-427

\begin{tabular}{l|l}
\hline DOI: $10.1159 / 000339035$ & $\begin{array}{l}\text { C } 2012 \text { S. Karger AG, Basel } \\
\text { www.karger.com/cpb }\end{array}$
\end{tabular}

Published online: July 06, 2012

www.karger.com/cpb

Giovannetti/Gambardella/Pietraforte et al.: Erythrocyte Fate in Scleroderma

\begin{tabular}{ccccc}
\hline & Healthy donors & All SSc patients & lc-SSc patients & dc-SSc patients \\
\hline Glycophorin A (MFI) & $101 \pm 15$ & $76 \pm 7^{*}$ & $71 \pm 5^{*}$ & $87 \pm 9$ \\
Band 3 (MFI) & $165 \pm 10$ & $73 \pm 8^{* *}$ & $64 \pm 5^{* *}$ & $98 \pm 4^{* *}$ \\
CD47 (MFI) & $280 \pm 5$ & $264 \pm 2$ & $193 \pm 4^{* *}$ & $334 \pm 2^{* *}$ \\
PS externalization (\%) & $0.2 \pm 0.04$ & $0.6 \pm 0.08^{* *}$ & $0.6 \pm 0.03^{* *}$ & $0.7 \pm 0.05^{* *}$ \\
\hline
\end{tabular}

Table 3. Molecules involved in red blood cell aging. MFI= median fluorescence intensity. To compare healthy donors with the two subgroups of SSc patients, one-way ANOVA followed by Tukey Test was used. We found significant differences between the three subgroups (Glycophorin A: $p=0.0151$; Band 3, CD47 and PS externalization: $\mathrm{p}<0.0001) .\left({ }^{*}\right) \mathrm{p}<0.05$ vs healthy donors; $\left({ }^{* *}\right) \mathrm{p}<0.0001$ vs healthy donors.

Fig. 2. NVC patterns and PS externalization in SSc patients. PS externalization at the RBC surface of SSc patients according to the different patterns of NVC (early, active and late). The non-parametric Kruskal-Wallis test showed statistically significant differences between the three subgroups $(\mathrm{p}=0.002)$. Responsible for this difference was the subgroup "late" as demonstrated by the Bonferroni post-hoc test ("late" vs "active" $\mathrm{p}=0.002$ and "late" vs "early" $\mathrm{p}<0.0001)$.

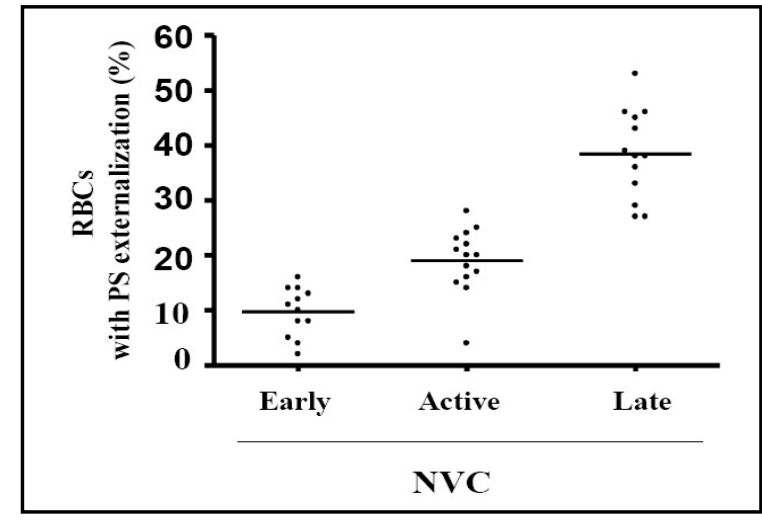

an integrin-associated protein that is known as thrombospondin receptor that acts as a "marker of self" [24]. The fourth is a phospholipid normally localized to the inner leaflet of the plasma membrane, which is externalized to the outer leaflet during cell remodeling leading to RBC aging and cell death [25]. The analyses of GA, band 3 and CD47, carried out by flow-cytometry, clearly demonstrated an altered expression of these proteins in RBCs from SSc patients with respect to those from healthy donors. In particular, we found a significant decrease $(p<0.05)$ of GA expression only in RBCs from lc-SSc patients. As concerns band 3, its expression was found significantly decreased $(\mathrm{p}<0.01)$ in RBCs from both lc-SSc and dc-SSc patients. Conversely, the analysis of CD47 expression demonstrated a significant decrease $(\mathrm{p}<0.01)$ in lc-SSc patients and a significant increase $(\mathrm{p}<0.01)$ in RBCs from dc-SSc patients (Table 3 and Fig. 1A, C, E). These results were confirmed by immunofluorescence analyses, which showed a dramatic rearrangement and redistribution of these molecules (Fig $1 B, D$, F). Moreover, as concerns PS externalization, a significantly $(\mathrm{p}<0.01)$ increased percentage of RBCs showing translocation of this phospholipid at the outer plasma membrane leaflet was detected in cells from SSc patients (both lc-SSc and dc-SSc) with respect to those from healthy donors (Table 3). To verify if the appearance of aging markers was accompanied by changes in mean corpuscular volume (MCV) and mean cell hemoglobin concentration (MCHC), further investigations were performed. For both MCV and MCHC, values detected were in the normal range (normal range = MCV: 80-100 fL; MCHC: $32-36 \mathrm{~g} / \mathrm{dl}$; dc-SSc $=\mathrm{MCV}$ : $86 \pm 2$ fL; MCHC: $30 \pm 2$ g/dl; lc-SSc = MCV: $87 \pm 5$ fL; MCHC: $31 \pm 3$ g/dl).

\section{Relationships between clinical features and RBC morphology}

We finally evaluated whether microvascular damage (i.e. NVC) was associated with RBC changes in SSc patients by using the Kruskal-Wallis one-way analysis of variance followed by the post hoc Bonferroni test. The results showed that there was no significant relationship between NVC and ROS production $(\mathrm{p}=0.536)$ as well as between NVC and the decreased expression for GA $(p=0.071)$. A significant association was observed between NVC and PS externalization $(p=0.002)$. In particular, the Bonferroni post hoc test showed 


\section{Cellular Physiology Cell Physiol Biochem 2012;30:418-427 \\ \begin{tabular}{ll|l} 
and Biochemistry & DOI: 10.1159/000339035 & P) 2012 S. Karger AG, Basel \\
\cline { 2 - 3 } Published online: July 06, 2012 & Gww.karger.com/cpb \\
\cline { 2 - 3 } & Giovannetti/Gambardella/Pietraforte et al.: Erythrocyte Fate in Scleroderma
\end{tabular}}

a significant difference between the subgroup "late" and both the "active" ( $\mathrm{p}=0.002)$ and "early" ( $<<0.0001$ ) subgroups (Fig. 2). Interestingly, Spearman rank correlation analysis revealed a significant positive correlation between PS externalization and mRTSS ( $p<0.0001$; $\rho=0.84)$, DSS $(p=0.004 ; \rho=0.62)$ and $\operatorname{sDAS}(p<0.0001 ; \rho=0.86)$.

\section{Discussion}

Systemic Sclerosis (SSc) is a multisystem disease that affects the skin and internal organs (lungs, heart, kidney and gut) eventually leading to tissue fibrosis. Microvascular endothelial injury, a vasospastic tendency with inadequate vasodilatory response and vessels changes, including alteration of the coagulative/fibrinolytic system and proliferation of intimal cells leading to obliteration of the microvasculature, are primary events that occur early in SSc [26]. Among the different factors that may contribute to the inflammation and derangement of the endothelium, thus favoring disease progression, is the stress induced on the endothelium by oxygen free radicals and reactive nitrogen species $[27,28]$. This has led to hypothesize that in SSc patients chronic oxidative imbalance could be associated with endothelial cell ageing and damage that impairs vascular supply, leading to tissue hypoxia and promoting inflammation and fibrosis $[28,29]$.

In this paper, we investigated if oxidative imbalance, which occurs in SSc patients, affects RBC homeostasis. Because of its high iron concentration $(\sim 20 \mathrm{mM})$, the RBC can be considered as an "iron mine" but, paradoxically, it also represents one of the major components of blood antioxidant capacity and one of the cells with higher resistance to oxidative stress [29]. In fact, crossing inflammed areas, the RBCs can contribute to detoxify reactive oxygen and nitrogen species thus rescuing or partially "protecting" cells (e.g. endothelial cells). Completely different may be the situation when the RBC crosses a tissue where an intense production of reactive oxygen/nitrogen species occurs. Under these conditions, the RBCs may accumulate oxidative damage and act as pro-oxidant "bullets" capable of modifying the behavior and fate of endothelial cells [30].

Our results suggest that the systemic oxidative imbalance occurring in SSc patients could cause a plethora of changes in RBCs, including cytoskeleton oxidative denaturation and derangement, and loss of lipid asymmetry. These changes can result in alterations of their adhesive properties and aggregability as well as in their deformability, an essential rheological property. Important differences were found by analyzing separately RBCs from lc-SSc and dc-SSc patients. In particular, we found that: i) in RBCs from lc-SSc patients the total thiol content significantly decreased and the expression of GA, band 3 and CD47 at the cell surface was also significantly decreased. By contrast, ii) in RBCs from dc-SSc patients, ROS levels and CD47 expression significantly increased. On the basis of these data we can suggest that RBCs from lc-SSc patients could undergo to mild redox alteration that, in turn, could induce premature aging and consequent removal of RBCs from circulation. Conversely, RBCs from dc-SSc patients over-expressing CD 47, could "escape" from their phagocytosis. In fact, it has been demonstrated that the interaction between the CD47 molecules on normal RBCs and signal regulatory protein $\alpha$ receptors on macrophages sends negative signals to macrophages protecting RBCs from phagocytosis [31]. This could be at least partially related to the previously suggested implication of CD47 in autoimmune disease susceptibility and progression [32, 33]. In line with this, decreased expression of CD47 on RBCs from lc-SSc (68\% lower than in the RBCs from HD), could be one of the mechanisms that regulate the removal by phagocytosis of senescent RBCs in these patients, contributing to disease progression. Conversely, RBCs from dc-SSc patients, expressing more CD47 molecules at their surface, could escape phagocytosis and, remaining in circulation, could adhere to the endothelial cells, forming aggregates and promoting thrombotic events [34]. In support of this hypothesis PS externalization can play an important role. In fact, the detection of high levels of PS on the outer leaflet of the RBC plasma membrane $(0,7 \%$ of RBCs from dc-SSc patients) could contribute to cell-cell aggregation, e.g. between RBCs and endothelial cells (EC). In fact, it has been reported that PS, expressed on RBC surface, could act as a potent 


\section{Cellular Physiology Cell Physiol Biochem 2012;30:418-427 \\ \begin{tabular}{ll|l} 
and Biochemistry & $\begin{array}{l}\text { DOI: 10.1159/000339035 } \\
\text { Published online: July 06, 2012 }\end{array}$ & $\begin{array}{l}\text { O 2012 S. Karger AG, Basel } \\
\text { www.karger.com/cpb }\end{array}$ \\
\hline Giovannetti/Gambardella/Pietraforte et al.: Erythrocyte Fate in Scleroderma
\end{tabular}}

mediator of RBC/EC adhesion and facilitate thrombus formation [34]. Furthermore, these data are in line with previously demonstrated presence of anti-PS/Protrombin Abs in the blood plasma from SSc patients [35]. In fact, we can not rule out the possibility that the appearance of these autoantibodies could somewhat be related to the expression of PS at the RBC surface. Finally, we found a highly significant correlation between PS levels at the RBC surface and the NVC evaluations. This observation is in line with the correlation recently demonstrated, in SSc patients, between lymphocyte undergoing apoptosis, which expose PS at their surface, and disease activity [36]. Therefore, if the severity of SSc associates with increasing levels of PS externalization on both RBCs and lymphocytes, it is tempting to speculate a therapeutic usage of antiapoptotic agents for patients with SSc. In fact, agents such as $\mathrm{N}$-acetylcysteine, which acts as antioxidant and is known for its antiapoptotic properties [37], already represents a useful therapeutic choice in the clinical management of SSc during the active phases of the disease [38, 39].

In conclusion, our data, showing for the first time a striking correlation between eryptosis, the apoptosis of RBC [40], and disease severity as measured by NVC, add a useful piece of information to the complex scenario of SSc pathogenesis and suggest RBCs as novel candidate biomarkers for the monitoring of this rare human disease.

\section{Abbreviations}

dc-SSc (diffuse cutaneous systemic sclerosis); DSS (Scleroderma Disease Severity Scale); EC (endothelial cells); ESR (erythrocyte sedimentation rate); GA (glycophorin A); HD (healthy donors); lc-SSc (limited cutaneous systemic sclerosis); LDL (low-density lipoprotein); MCHC (mean cell hemoglobin concentration); MCV (mean corpuscular volume); mRTSS (modified Rodnan Total Skin Score); NVC (nailfold videocapillaroscopy); PAH (pulmonary arterial hypertension); PS (phosphatidylserine); RBC (red blood cell); ROS (reactive oxygen species); RP (Raynaud's phenomenon); SDAS (Scleroderma Disease Activity Score); SSc (systemic sclerosis).

\section{Acknowledgements}

We thank Daniela Farinelli for her precious support. This work has partially been supported by grant from Ministero della Sanita' to W.M.

\section{Conflict of Interest Disclosure}

No financial interest/relationships with financial interest relating to the topic of this article have to be declared.

\section{References}

1 Clements PJ: Systemic sclerosis (scleroderma) and related disorders: clinical aspects. Bailleres Best Pract Res Clin Rheum 2000;14:1-16.

2 Merkel PA, Herlyn K, Martin RW, Anderson JJ, Mayes MD, Bell P, Korn JH, Simms RW, Csuka ME, Medsger TA Jr, Rothfield NF, Ellman MH, Collier DH, Weinstein A, Furst DE, Jiménez SA, White B, Seibold JR, Wigley FM; Scleroderma Clinical Trials Consortium: Measuring disease activity and functional status in patients with scleroderma and Raynaud's phenomenon. Arthritis Rheum 2002;46:2410-2420.

3 LeRoy EC, Black C, Fleischmajer R, Jablonska S, Krieg T, Medsger TA Jr, Rowell N, Wollheim F: Scleroderma (systemic sclerosis): classification, subsets and pathogenesis. J Rheumatol 1988;15:202-205. 


\section{Cellular Physiology Cell Physiol Biochem 2012;30:418-427 \\ \begin{tabular}{ll|l} 
and BiOChemistry & $\begin{array}{l}\text { DOI: 10.1159/000339035 } \\
\text { Published online: July 06, 2012 }\end{array}$ & $\begin{array}{l}\text { (c) 2012 S. Karger AG, Basel } \\
\text { www.karger.com/cpb }\end{array}$ \\
\cline { 2 - 3 } Giovannetti/Gambardella/Pietraforte et al.: Erythrocyte Fate in Scleroderma
\end{tabular}}

4 Hachulla E, Launay D, Mouthon L, Sitbon O, Berezne A, Guillevin L, Hatron PY, Simonneau G, Clerson P, Humbert M: French PAH-SSc Network Is pulmonary arterial hypertension really a late complication of systemic sclerosis? Chest 2009;136:1211-1219.

$5 \quad$ Krieg T, Takehara K: Skin disease: a cardinal feature of systemic sclerosis. Rheumatology 2009;48:14-18.

6 Yamamoto T: Autoimmune mechanisms of scleroderma and a role of oxidative stress. Self Nonself 2011;2:410.

7 Sambo P, Jannino L, Candela M, Salvi A, Donini M, Dusi S: Monocytes of patients with systemic sclerosis (scleroderma) spontaneously release in vitro increased amounts of superoxide anion. J Invest Dermatol 1999;112:78-84.

8 Esterbauer H, Dieber-Rothender M, Maeg G, Striegl G, Jurges G: Biochemical structural and functional properties of oxidised low-density lipoprotein. Chem Res Toxicol 1990;3:77-92.

9 Pratico` D: Antioxidants and endothelium protection. Atherosclerosis 2005;181:215-224.

10 Yarnold J, Brotons MC: Pathogenetic mechanisms in radiation fibrosis. Radiother Oncol 2010;97:149-161.

11 Jia Y, Wood F, Menu P, Faivre B, Caron A, Alayash AI: Oxygen binding and oxidation reactions of human hemoglobin conjugated to carboxylate dextran. Biochim Biophys Acta 2004;1672:164-173.

12 Cosby K, Partovi KS, Crawford JH, Patel RP, Reiter CD, Martyr S, Yang BK, Waclawiw MA, Zalos G, Xu X, Huang KT, Shields H, Kim-Shapiro DB, Schechter AN, Cannon RO 3rd, Gladwin MT: Nitrite reduction to nitric oxide by deoxyhemoglobin vasodilates the human circulation. Nat Med 2003;9:1498-1505.

13 Minetti M, Agati L, Malorni W: The microenvironment can shift erythrocytes from a friendly to a harmful behavior: pathogenetic implications for vascular diseases. Cardiovasc Res 2007;75:21-28.

14 Baskurt OK, Meiselman HJ: Red blood cell “aggregability”. Clin Hemorheol Microcirc 2009;43:353-354.

15 Yedgar S, Kaul DK, Barshtein G: RBC adhesion to vascular endothelial cells: more potent than RBC aggregation in inducing circulatory disorders. Microcirculation 2008;15:581-583.

16 Subcommittee for scleroderma criteria of the American Rheumatism Association Diagnostic and Therapeutic Criteria Committee Arthritis Rheum. Preliminary criteria for the classification of systemic sclerosis (scleroderma). 1980;23:581-590.

17 Clements P, Lachenbruch P, Siebold J, White B, Weiner S, Martin R, Weinstein A, Weisman M, Mayes M, Collier D: Inter and intraobserver variability of total skin thickness score (modified Rodnan TSS) in systemic sclerosis. J Rheumatol 1995;22:1281-1285.

18 Valentini G, D’Angelo S, Della Rossa A, Bencivelli W, Bombardieri S: European Scleroderma Study Group to define disease activity criteria for systemic sclerosis. IV. Assessment of skin thickening by modified Rodnan skin score. Ann Rheum Dis 2003;62:904-905.

19 Valentini G, Della Rossa A, Bombardieri S, Bencivelli W, Silman AJ, D’Angelo S, Cerinic MM, Belch JF, Black CM, Bruhlmann P, Czirják L, De Luca A, Drosos AA, Ferri C, Gabrielli A, Giacomelli R, Hayem G, Inanc M, McHugh NJ, Nielsen H, Rosada M, Scorza R, Stork J, Sysa A, van den Hoogen FH, Vlachoyiannopoulos PJ: European multicentre study to define disease activity criteria for systemic sclerosis. II. Identification of disease activity variables and development of preliminary activity indexes. Ann Rheum Dis 2001;60:592-598.

20 Medsger TA Jr, Silman AJ, Steen VD, Black CM, Akesson A, Bacon PA, Harris CA, Jablonska S, Jayson MI, Jimenez SA, Krieg T, Leroy EC, Maddison PJ, Russell ML, Schachter RK, Wollheim FA, Zacharaie H: A disease severity scale for systemic sclerosis: development and testing. J Rheumatol 1999;26:2159-2167.

21 Cutolo M, Sulli A, Pizzorni C, Accardo S: Nailfold videocapillaroscopy assessment of microvascular damage in systemic sclerosis. J Rheumatol 2000;27:155-160.

22 Caprari P, Bozzi A, Malorni W, Bottini A, Iosi F, Santini MT, Salvati AM: Junctional sites of erythrocyte skeletal proteins are specific targets of tert-butylhydroperoxide oxidative damage. Chem Biol Interact 1995;943:243258.

23 Matarrese P, Straface E, Pietraforte D, Gambardella L, Vona R, Maccaglia A: Peroxynitrite induces senescence and apoptosis of red blood cells through the activation of aspartyl and cysteinyl proteases. FASEB J 2005;19:416-418.

24 Khandelwal S, van Rooijen N, Saxena RK: Reduced expression of CD47 during murine red blood cell (RBC) senescence and its role in RBC clearance from the circulation. Transfusion 2007;47:1725-1732.

25 Mandal D, Mazumder A, Das P, Kundu M, Basu J: Fas-, caspase 8-, and caspase 3-dependent signaling regulates the activity of the aminophospholipid translocase and phosphatidylserine externalization in human erythrocytes. J Biol Chem 2005;280:39460-39467.

26 Manetti M, Guiducci S, Ibba-Manneschi L, Matucci-Cerinic M: Mechanisms in the loss of capillaries in systemic sclerosis: angiogenesis versus vasculogenesis. J Cell Mol Med 2010;14:1241-1254. 


\section{Cellular Physiology \\ Cell Physiol Biochem 2012;30:418-427 and Biochemistry

\begin{tabular}{l|l}
\hline DOI: $10.1159 / 000339035$ & C 2012 S. Karger AG, Basel \\
Published online: July 06, 2012 & www.karger.com/cpb
\end{tabular} \\ Giovannetti/Gambardella/Pietraforte et al.: Erythrocyte Fate in Scleroderma}

27 Konttinen YT, Mackiewicz Z, Ruuttila P, Ceponis A, Sukura A, Povilenaite D, Hukkanen M, Virtanen I: Vascular damage and lack of angiogenesis in systemic sclerosis skin. Clin Rheumatol 2003;22:196-202.

28 Förstermann U: Nitric oxide and oxidative stress in vascular disease. Pflugers Arch 2010;459:923-939.

29 Minetti M, Malorni W: Redox control of red blood cell biology: the red blood cell as a target and source of prooxidant species. Antioxid Redox Signal 2006;8:1165-1169.

30 Buehler PW, Alayash AI: Oxygen sensing in the circulation: "cross talk" between red blood cells and the vasculature. Antioxid Redox Signal 2004;6:1000-1010.

31 Oldenborg PA, Zheleznyak A, Fang YF, Lagenaur CF, Gresham HD, Lindberg FP: Role of CD47 as a marker of self on red blood cells. Science 2000;288:2051-2054.

32 Oldenborg PA: Role of CD47 in erythroid cells and in autoimmunity. Leuk Lymphoma 2004;45:1319-1327.

33 Dugas V, Beauchamp C, Chabot-Roy G, Hillhouse EE, Lesage S: Implication of the CD47 pathway in autoimmune diabetes. J Autoimmun 2010;35:23-32.

34 Yedgar S, Koshkaryev A, Barshtein G: The red blood cell in vascular occlusion. Pathophysiol Haemost Thromb 2002;32:263-268.

35 Arnout J: The pathogenesis of the antiphospholipid syndrome: a hypothesis based on parallelisms with heparin-induced thrombocytopenia. Thromb Haemost 1996;75:536-541.

36 Giovannetti A, Rosato E, Renzi C, Maselli A, Gambardella L, Giammarioli AM, Palange P, Paoletti P, Pisarri S, Salsano F, Malorni W, Pierdominici M: Analyses of T cell phenotype and function reveal an altered T cell homeostasis in systemic sclerosis. Correlations with disease severity and phenotypes. Clin Immunol 2010;137:122-133.

37 Lucantoni G, Pietraforte D, Matarrese P, Gambardella L, Metere A, Paone G, Bianchi EL, Straface E: The red blood cell as a biosensor for monitoring oxidative imbalance in chronic obstructive pulmonary disease: an ex vivo and in vitro study. Antioxid Redox Signal. 2006;8:1171-1182.

38 Rosato E, Borghese F, Pisarri S, Salsano F: The treatment with N-acetylcysteine of Raynaud's phenomenon and ischemic ulcers therapy in sclerodermic patients: a prospective observational study of 50 patients. Clin Rheumatol 2009;28:1379-1384.

39 Del Papa N, Quirici N, Scavullo C, Gianelli U, Corti L, Vitali C, Ferri C, Giuggioli D, Manfredi A, Maglione W, Onida F, Colaci M, Bosari S, Lambertenghi Deliliers G: Antiendothelial cell antibodies induce apoptosis of bone marrow endothelial progenitors in systemic sclerosis. J Rheumatol 2010;37:2053-2063.

40 Lang F, Lang KS, Lang PA, Huber SM, Wieder T: Mechanisms and significance of eryptosis. Antioxid Redox Signal 2006;8:1183-1192. 